\title{
Special Issue on Advances in Channel Coding
}

\author{
Erdal Arıkan, Michael Lentmaier, and Guido Montorsi
}

Since the invention of turbo codes in 1993 there has been an enormous interest and progress in the field of capacity approaching code constructions. Many classical constructions have been replaced by newer, better performing codes with feasible decoding complexity. Most of these modern code constructions, such as turbo codes, Gallager's low-density parity-check (LDPC) codes and their generalizations, can be modeled by sparse graphical models. Spatial coupling of sparse graphical models has in the last years attracted a lot of interest due to the threshold saturation phenomenon, which leads to capacity achieving performance with iterative message passing decoding. Polar codes are a recently discovered class of capacity achieving codes that are formed by an explicit construction based on a phenomenon called channel polarization. These codes, too, have various low-complexity decoding algorithms based on message passing on a sparse graph that has a recursive structure similar to that of fast transforms in signal processing.

Despite the enormous advances in channel coding in the last two decades, the state-of-the-art in this area is far from meeting the challenges of the near-future communication systems in terms of throughput, performance, robustness, flexibility and energy consumption. To give one example, it is unclear how current forward errorcorrection coding (FEC) techniques will scale to the $100+\mathrm{GB} / \mathrm{s}$ data rates foreseen for wireless backhaul communications in the near future. New code constructions and algorithms as well as novel concepts may have to replace the techniques that we use today. The purpose of this special issue is to address the challenges of future channel coding schemes.

The paper "Challenges and Some New Directions in Channel Coding" by Erdal Arıkan, Najeeb ul Hassan, Michael Lentmaier, Guido Montorsi and Jossy Sayir, presents a survey of three recent research areas in channel coding, which have been investigated within the European Union FP7 Network of Excellence NEWCOM\#: Spatially coupled LDPC codes, non-binary LDPC codes, and polar coding. It is demonstrated that coding is still an active area of research with many challenges remaining and a number of promising innovations are on their way to maturing into technological advances in the coming years. Efficient decoding of LDPC codes is the topic of the paper "Iterative Reliability-based Modified Majority-Logic Decoding for Structured Binary LDPC Codes" by Haiqiang Chen, Lingshan Luo, Youming Sun, Xiangcheng Li, Haibin Wan, Liping Luo and Tuanfa Qin. The main feature of the presented algorithm is to provide efficient trade-offs between decoding performance and computational complexity. The main computational steps of the presented algorithm include only binary logic and integer operations and with some computer simulations it is demonstrated that the performance gap between the sum-product algorithm and the presented algorithm is less than $0.6 \mathrm{~dB}$. Coding techniques for cooperative communications are addressed in the paper "Rate-Compatible LDPC Codes based on PEG Algorithm for Relay Communication Systems" by Yangzhao Zhou, Xueqin Jiang, and Moon Ho Lee. A class of LDPC codes for relay 
communication systems is considered that is based on extending so-called root-LDPC codes, which are known to achieve full diversity on block-fading channels when decoded iteratively. A modified PEG algorithm is proposed, imposing some construction constraints that lead to improved performance compared to previous results in the literature.

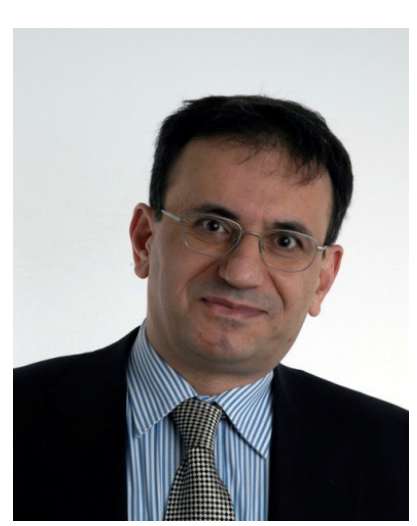

Erdal Arıkan was born in Ankara, Turkey, in 1958. He received the B.S. degree from the California Institute of Technology, Pasadena, in 1981 and the S.M. and Ph.D. degrees from the Massachusetts Institute of Technology, Cambridge, MA, in 1982 and 1985, respectively, all in electrical engineering. Since 1987 he has been with the Electrical-Electronics Engineering Department of Bilkent University, Ankara, Turkey, where he is presently a Professor. His research Interests include coding techniques, coding theory, and Shannon theory. He has received many awards including the IEEE Information Theory Society Paper Award (2010) and the IEEE W.R.G. Baker Award (2013) for his paper "Channel Polarization: A Method for Constructing CapacityAchieving Codes for Symmetric Binary-Input Memoryless Channels”.

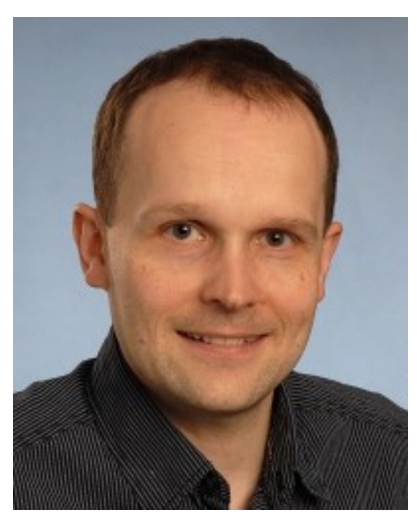

Michael Lentmaier received the Dipl.-Ing. degree in electrical engineering from University of Ulm, Germany in 1998, and the Ph.D. degree in telecommunication theory from Lund University, Sweden in 2003. He then worked as a Post-Doctoral Research Associate at University of Notre Dame, Indiana and at University of Ulm. From 2005 to 2007 he was with the Institute of Communications and Navigation of the German Aerospace Center (DLR) in Oberpfaffenhofen, where he worked on signal processing techniques in satellite navigation receivers. From 2008 to 2012 he was a senior researcher and lecturer at the Vodafone Chair Mobile Communications Systems at TU Dresden, where he was heading the Algorithms and Coding research group. Since January 2013 he is an Associate Professor at the Department of Electrical and Information Technology at Lund University. His research interests include design and analysis of coding systems, graph based iterative algorithms and Bayesian methods applied to decoding, detection and estimation in communication systems. He is a senior member of the IEEE and served as an editor for IEEE Communications Letters from 2010 to 2013 and IEEE Transactions on Communications since 2014. He was awarded the Communications Society \& Information Theory Society Joint Paper Award (2012) for his paper "Iterative Decoding Threshold Analysis for LDPC Convolutional Codes." 


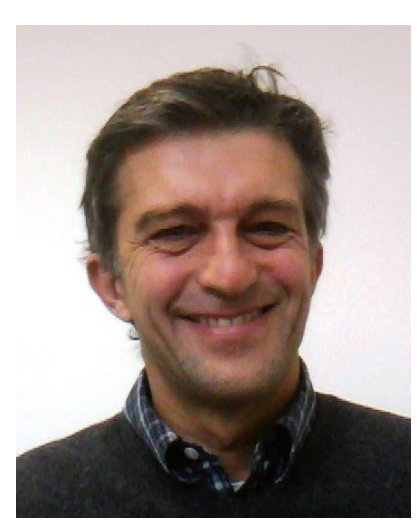

Guido Montorsi is a Full Professor at the Politecnico di Torino, Italy, where he also received the Ph.D. degree in telecommunications from the Dipartimento di Elettronica. His master thesis concerned the study and design of coding schemes for HDTV, developed at the RAI Research Center, Turin. Professor Montorsi has been at the Department of Electrical Engineering at the Rensselaer Polytechnic Institute, Troy, NY, USA, and later at Sequoia Communications, CA, USA, working with the innovative design and implementation of a 3rd generation WCDMA receiver. His interests are in the area of channel coding and wireless communications, particularly on the analysis and design of concatenated coding schemes and study of iterative decoding strategies. 